Onkologie 1993;16:1

\title{
Impressum, Vol. 16, No. 1, 1993
}

Offizielles Organ der Deutschen Gesellschaft für Hämatologie und Onkologie, der Österreichischen Gesellschaft für Hämatologie und Onkologie, der Österreichischen Krebsgesellschaft - Krebsliga unter Fortführung der «Österreichischen Zeitung für Onkologie» Schriftleitung

H. Huber, Wien

W. Queißer, Mannheim

Fachschriftleitung

W. E. Berdel, Berlin E. Dühmke, Göttingen N. Jaeger, Hildesheim M. Kaufmann, Heidelberg P. Schlag, Berlin

Wïssenschaftlicher Beirat

L. Bergmann, Frankfurt/M. V. Budach, Essen V. Diehl, Köln C. Dittrich, Wien P. Drings, Heidelberg L. Edler, Heidelberg H. H. Fiebig, Freiburg

C. Glanzmann, Zurich

R. Greiner, Bern

R. Hartenstein, München K. Havemann, Marburg K. P. Hellriegel, Berlin R. Herrmann, Basel

H.W. von Heyden, Einbeck

D. Hoelzer, Frankfurt/M.

K. Höffken, Jena

J. H. Holzner, Wien H. J. Illiger, Oldenburg R. Jakesz, Wien

U. R. Kleeberg, Hamburg

B. Kornhuber, Frankfurt/M.

R. Kreienberg, Ulm

M. Lahousen, Graz

H. Löffler, Kiel

H. Ludwig, Wien

P. Lukas, München

U. Mohr, Hannover

G. A. Nagel, Uitikon-Zürich

A. Neiß, Innsbruck

A. Pfleiderer, Freiburg

F. Porzsolt, Ulm

K. Possinger, München H.-J. Schmoll, Hannover M. Schroder, Kassel S. Seeber, Essen P. Sevelda, Wien

B. Thürlimann, St. Gallen

H. W Waclawiczek, Salzburg

M. Wannenmacher, Heidelberg

H. J. Weh, Hamburg

W. Wilmanns, München 
Die Zeitschrift erscheint zweimonatlich; pro Jahr erscheint 1 Band zu je 6 Heften.

Bezugspreis für Jahrgang 16,1993, DM 159,-/öS 1113-/SFr 124,-. 1 Einzelheft kostet DM 30 -/öS 210 -/SFr 23,-, einschließlich MwSt., zuzüglich Postgebühren.

Der Abonnementpreis ist im voraus zahlbar. Das Abonnement der Zeitschrift läuft weiter, wenn es nicht spätestens 4 Wochen vor Abschluß eines Bandes abbestellt wird.

Abonnementbestellungen können bei jeder Buchhandlung oder direkt beim Verlag aufgegeben werden:

Bundesrepublik Deutschland/Österreich: S. Karger GmbH, Lörracher Str. 16 a, W-7800 Freiburg, Telefon (0761) 452070, Telefax (0761) 452 0714, Postgiro München 40080-807

Schweiz: S. Karger AG, Allschwilerstr. 10, Postfach, CH-4009 Basel, Telefon (061) 3061111, Telex 62652 CH, Telefax (061) 3061234

Anzeigen

S. Karger Verlag für Medizin und Naturwissenschaften GmbH, Lörracher Str. 16a, W-7800 Freiburg, Telefon (0761) 452070.

Gültig ist die Preisliste Nr. 7 vom 1. Januar 1993.

Für den Inhalt außerhalb des redaktionellen Teiles (insbesondere Anzeigen, Industrieinformationen, Pressezitate und Kongreßinformationen) übernehmen Schriftleitung, Beirat und Verlag keine Gewähr.

Eine Markenbezeichnung kann warenzeichenrechtlich geschützt sein, auch wenn bei ihrer Verwendung in dieser Zeitschrift das Zeichen ${ }^{\circledR}$ oder ein anderer Hinweis auf etwa bestehende Schutzrechte fehlen sollte. Für Satzfehler, insbesondere bei Dosierungsangaben, wird keine Gewähr übernommen.

Die Zeitschrift sowie alle in ihr enthaltenen einzelnen Beiträge und Abbildungen sind urheberrechtlich geschützt. Jede Verwertung, die nicht ausdrücklich vom Ur-heberrechtsgesetz zugelassen ist, bedarf der vorherigen Zustimmung des Verlags. Das gilt insbesondere für Vervielfältigungen, Bearbeitungen, Übersetzungen, Mi-kroverfilmungen und die Einspeicherung und Verarbeitung in elektronischen Sy-stemen. Fotokopien dürfen nur für den persönlichen Gebrauch als Einzelkopien hergestellt werden. Jede im Bereich eines gewerblichen Unternehmens zulässig hergestellte oder benutzte Kopie dient gewerblichen Zwecken gem. § 54(2) UrhG und verpflichtet zur Gebührenzahlung an die Verwertungsgesellschaft WORT, Abt. VG Wissenschaft, Goethestraße 49, W-8000 München 2.

(C) Copyright 1993 by S. Karger

Verlag für Medizin und Naturwissenschaften GmbH, Lörracher Str. 16 a

W-7800 Freiburg (BRD)

Verlagsleitung: Steven Karger

Presserechtlich verantwortlich: Georg Brunner Redaktionsassistenz: Martina Zeller

Anzeigenverwaltung: Christiane Opitz

Satz und Druck: Walter Biering GmbH Grafischer Betrieb Freisinger Landstraße 21 W-8000

München 45 (BRD)

KARGER 